\title{
Structural heart disease, not right ventricular pacing site, determines QRS duration during right ventricular pacing
}

\author{
Michio Ogano ${ }^{1}$, Ippei Tsuboi ${ }^{1}$, Yu-ki Iwasaki², Jun Tanabe ${ }^{1}$, and Wataru Shimizu ${ }^{2}$ \\ ${ }^{1}$ Shizuoka Medical Center \\ ${ }^{2}$ Nippon Medical School
}

September 11, 2020

\begin{abstract}
Introduction: Right ventricular (RV) pacing causes changes in the heart's electrical and mechanical activation patterns. QRS duration is a useful surrogate marker of electrical dyssynchrony; longer QRS duration during RV pacing indicates poor prognosis. However, the mechanisms underlying longer QRS duration during RV pacing remain unclear; hence, we investigated factors predicting QRS prolongation during RV pacing. Methods and Results: We enrolled 211 patients who underwent catheter ablation for supraventricular tachyarrhythmia and showed no bundle-branch-block. Three-dimensional mapping for QRS duration during RV pacing from the RV outflow to RV apex was performed, and the difference in QRS duration was analyzed. The predisposing factors causing QRS $>160 \mathrm{~ms}$ during RV apical pacing were also analyzed. QRS durations at baseline and during RV pacing from the RV outflow and at RV apex were $85.0 \pm 7.5 \mathrm{~ms}, 163.7 \pm 17.1 \mathrm{~ms}$, and $156.2 \pm 16.1 \mathrm{~ms}$, respectively. With respect to QRS duration, there was a significant correlation between RV outflow and RV apical pacing $(\mathrm{r}=0.658, \mathrm{p}<0.001)$. The difference in QRS duration between RV outflow and apex in each patient was only $12.5 \pm 10.4 \mathrm{~ms}$. Logistic multivariable regression analysis identified baseline QRS duration [odds ratio (OR) 1.24, 95\% confidence interval (CI) 1.15 to 1.33, p <0.01], interventricular septum thickness (OR 1.20, 95\% CI 1.02-1.40, p=0.025), left atrial diameter (OR 1.08, 95\% CI 1.01-1.16, p=0.024), and E/e' (OR 1.23, 95\% CI 1.12-1.35, p<0.01) as significant predictors of prolonged QRS duration during RV apical pacing. Conclusion: QRS duration during RV pacing largely depends not on the pacing site, but on underlying structural heart diseases.
\end{abstract}

\section{Structural heart disease, not right ventricular pacing site, determines QRS duration during} right ventricular pacing

Michio Ogano, MD+, Ippei Tsuboi, MD++, Yu-ki Iwasaki, MD§, Jun Tanabe, MD+, Wataru Shimizu MDSS

+ Department of Cardiovascular Medicine

Shizuoka Medical Center, 762-1 Nagasawa, Shimizu, Sunto Shizuoka 4110906, Japan

++ Department of Cardiovascular Medicine

Nippon Medical School Musasikosugi hospital, 1-396 Kosugi-cho, Nakahar-ku, Kawasaki, Kanagawa 2118533, Japan

SSDepartment of Cardiovascular Medicine,

Nippon Medical School, 1-1-5 Sendagi, Bunkyo Tokyo 1138603, Japan

Funding: This research did not receive any specific grant from funding agencies in the public, commercial, or not-for-profit sectors.

Conflicts of interest: All authors have no conflicts of interest.

Address for correspondence and reprint requests: Michio Ogano, MD 
Division of Cardiovascular Medicine, Shizuoka Medical Center,

762-1 Nagasawa, Shimizu, Sunto Shizuoka 4110906, Japan

Phone: +81-5-5975-2000, Fax: +81-5-5975-1999, E-mail:m-ogano@nms.ac.jp

Abstract

Introduction: Right ventricular (RV) pacing causes changes in the heart's electrical and mechanical activation patterns. QRS duration is a useful surrogate marker of electrical dyssynchrony; longer QRS duration during RV pacing indicates poor prognosis. However, the mechanisms underlying longer QRS duration during RV pacing remain unclear; hence, we investigated factors predicting QRS prolongation during RV pacing.

Methods and Results: We enrolled 211 patients who underwent catheter ablation for supraventricular tachyarrhythmia and showed no bundle-branch-block. Three-dimensional mapping for QRS duration during RV pacing from the RV outflow to RV apex was performed, and the difference in QRS duration was analyzed. The predisposing factors causing QRS >160 ms during RV apical pacing were also analyzed. QRS durations at baseline and during RV pacing from the RV outflow and at RV apex were 85.0+-7.5 ms, 163.7+-17.1 $\mathrm{ms}$, and 156.2+-16.1 ms, respectively. With respect to QRS duration, there was a significant correlation between RV outflow and RV apical pacing $(\mathrm{r}=0.658, \mathrm{p}<0.001)$. The difference in QRS duration between RV outflow and apex in each patient was only $12.5+-10.4 \mathrm{~ms}$. Logistic multivariable regression analysis identified baseline QRS duration [odds ratio (OR) 1.24, 95\% confidence interval (CI) 1.15 to $1.33, \mathrm{p}<0.01$ ], interventricular septum thickness (OR 1.20, 95\% CI 1.02-1.40, p=0.025), left atrial diameter $(\mathrm{OR} 1.08,95 \%$ CI 1.01-1.16, $\mathrm{p}=0.024$ ), and E/e' (OR 1.23, 95\% CI 1.12-1.35, $\mathrm{p}<0.01$ ) as significant predictors of prolonged QRS duration during RV apical pacing.

Conclusion: QRS duration during RV pacing largely depends not on the pacing site, but on underlying structural heart diseases.

Keywords: Pacing, QRS duration, Heart Failure, Right ventricle, Electrical dyssynchrony

Introduction

Right ventricular (RV) pacing results in non-physiological electrical myocardial conduction and mechanical left ventricular (LV) dyssynchrony ${ }^{1}$ and may cause LV systolic dysfunction following heart failure even in patients without pre-existing systolic dysfunction. ${ }^{2,3}$ The emergence of a permanent physiological form of pacing such as His bundle pacing $(\mathrm{HBP})^{4}$ or left bundle branch pacing ${ }^{5,6}$ as well as several beneficial data ${ }^{4-6}$ suggest an alternative pacing management compared to conventional RV pacing.

Although many patients may obtain much benefit from a physiological form of pacing, successful placement of the HBP lead is only possible in approximately $80 \%$ of cases, ${ }^{7,8}$ and lead placement is technically complex and has elevated capture thresholds. ${ }^{9}$ Therefore, RV pacing remains the standard of care in the pacing management by virtue of its accessibility and relative stability over time. An additional reason is that most patients can tolerate RV pacing without any cardiovascular events for a long period. ${ }^{10}$

Prolonged QRS duration on ECG is a simple marker of the quantity of non-physiological electrical myocardial conduction and is an independent predictor of cardiovascular events. ${ }^{11}$ Narrower QRS after cardiac resynchronization therapy (CRT) predicts a good CRT response. ${ }^{12}$ Furthermore, widening of the QRS duration after CRT has been reported as an independent predictor of mortality or progression to heart transplantation. ${ }^{13}$ Recently, QRS-based CRT optimization was reported to be effective. ${ }^{14}$

A prolonged QRS duration during RV pacing has a detrimental effect on long-term cardiac function. ${ }^{15}$ QRS duration during RVP is a useful marker to identify patients who are at risk of heart failure events. However, detailed background on differences in QRS duration during RV pacing between patients is lacking. Our objective in this study was to investigate the predictive factors of QRS prolongation during RV pacing.

Methods 


\section{Patients}

From a consecutive group of patients at a single hospital, 295 patients who had undergone radiofrequency ablation for supraventricular arrhythmias: atrial fibrillation, atrial flutter, atrial tachycardia, atrioventricular reentrant tachycardia, and atrioventricular nodal reentrant tachycardia were prospectively enrolled. Patients were excluded if they had bundle branch block or native QRS duration longer than 120 ms at baseline or if they had congenital heart disease, frequent ventricular premature contraction or tachycardia, severe heart failure (NYHA III or IV), or acute coronary syndrome.

The medical histories of all patients were analyzed, and all patients underwent a baseline physical examination, a conventional 12-lead body surface ECG, and echocardiography. Echocardiographic images were obtained with a $3.5 \mathrm{MHz}$ transducer in the left lateral decubitus position using a commercially available system (Vivid Eight, General Electric-Vingmed, Milwaukee, WI). Standard 2D triggered to the QRS complex was saved in cine-loop format for offline analysis (EchoPac 6.06, GE Medical Systems, Horten, Norway). Echocardiographic parameters included left ventricular end-systolic volume (LVESV), left ventricular enddiastolic volume (LVEDV), left ventricular ejection fraction (LVEF), intraventricular wall thickness (IVST), posterior wall thickness (PWT), left atrial diameter (LAD), and the E/e' and E/A ratios. Ischemic cardiomyopathy was defined in the presence of significant coronary artery disease ( $>75 \%$ stenosis in 1 or more of the major epicardial coronary arteries) and/or history of myocardial infarction or prior revascularization.

Antiarrhythmic drugs except beta-blockers were stopped $>5$ half-lives before the study. No patient received amiodarone in the 6 months before the study. All patients provided written informed consent to participate, and the study was approved by our institutional ethics committee (18-13) and conducted in accordance with the Declaration of Helsinki.

Mapping during RV pacing

All patients entered into the electrophysiological laboratory in a fasting state. The electrophysiological study and catheter ablation were performed under deep sedation with intravenous propofol, pentazocine, and hydroxyzine hydrochloride. After the conventional ablation procedure was completed, the electrophysiological study of RV pacing was performed. The RV around the septal site mainly at its apex and outflow tracts was mapped with a 7.5 Fr, $3.5 \mathrm{~mm}$ open-irrigated-tip contact force (CF)-sensing ablation catheter (Navistar ThermoCool, SmartTouch, Biosense Webster, Diamond Bar, CA). Cardiac pacing was performed with a programable stimulator with 5.0 V output. A three-dimensional mapping system (CARTO 3, Biosense Webster) was used in real time with electrophysiological information, color-coded and superimposed on the anatomic map. The initial timing of the map was programmed to the pacing artifact signal, and electroanatomical mapping was conducted based on QRS duration during RV pacing: from the pacing artifact to the end of the QRS wave (Figure 1). Around the His bundle or right bundle branch area, careful pacing was conducted, and pure selective pacing of the His bundle or right bundle branch (abrupt QRS narrowing compared neighboring QRS duration) was excluded from the analysis.

Surface ECG was continuously monitored and stored on a computer-based digital amplifier-recorder system with optical disk storage for offline analysis. The 12 leads of the surface ECG were displayed in vertical alignment on the screen. QRS duration was measured as the interval between the earliest deflection of the ventricular complex in any of the 12 simultaneous leads and the latest offset in any lead by two experienced investigators. Measurements were repeated at a screen velocity of $50 \mathrm{~mm} / \mathrm{s}$.

During point acquisition, endocardial contact was facilitated by CF-sensing and myocardium capture by pacing. The locations of the RV outflow tract and RV apical site were identified based on the created electroanatomical mapping and axis in frank leads: the inferior axis in the RV outflow tract and superior axis in the RV apical site. The QRS duration was defined as wide when it was longer than $160 \mathrm{~ms}$ during RV apical pacing, which had previously shown a detrimental effect on long-term cardiac function. ${ }^{14,16,17}$

Statistical analysis

Continuous data are expressed as mean and standard deviation (SD), and categorial data are summarized 
as frequencies and percentages. Fisher's exact test and unpaired Student's t test were used to compare categorical and continuous data, respectively. Correlation analysis was performed to compare QRS duration during RV pacing and continuous baseline variables using Spearman correlation coefficients.

Univariate and multivariate logistic regression analyses were performed to identify possible predictors for wide QRS duration. The following variables were investigated: sex, age, baseline QRS duration, atrial fibrillation, hypertension, heart failure, ischemic cardiomyopathy, LVEF, LVEDV, LVESV, IVST, PWD, LAD, E/e', A/E, and use of beta blockers. The backward elimination method was applied. The multivariate models to predict variables for wide QRS duration were selected based on the univariate test results $(\mathrm{p}<0.1)$. Possible collinearity among eligible variables was assessed using variance inflation factors with a threshold equal to 5. To avoid statistical coupling of variables, separate multivariate models were constructed that excluded parameters derived from one another. Harrell's c-statistics were calculated for the different models. The model with the marginally higher Harrell's c-statistics was selected. A two-tailed probability P-value of 0.05 indicated statistical significance. Statistical analyses were calculated using SPSS version 25 (IBM Corp., Armonk, NY).

Results

From November 2018 to October 2019, 295 patients were consecutively enrolled. Of these patients, 72 patients with bundle branch block, 5 with severe heart failure (NYHA $>$ III), 5 with hypertrophic cardiomyopathy, and 2 with congenital heart disease were excluded. Data of the remaining 211 patients were included in the analysis; 161 had atrial fibrillation, 12 had atrial flutter, 20 had atrioventricular nodal reentrant tachycardia, 10 had atrioventricular reentrant tachycardia, and 8 had atrial tachycardia.

The average QRS duration at baseline, during RV outflow pacing, and during RV apical pacing was 80.5+$7.5 \mathrm{~ms}, 163.7+-17.1 \mathrm{~ms}$, and $156.2+-7.5 \mathrm{~ms}$, respectively. Pure selective His bundle or right bundle branch capture was not observed during pacing around the His or right bundle branch areas. There was a significant correlation of QRS duration between RV outflow pacing and RV apical pacing $(\mathrm{r}=0.658, \mathrm{p}<0.001)$ (Figure 2 ), signifying that patients with longer QRS duration during RV outflow pacing were highly likely to have longer QRS duration during RV apical pacing. The duration of QRS did not depend on the RV pacing site in each patient. The difference in QRS duration between RV outflow pacing and RV apical pacing in each patient was only $12.5+-10.4 \mathrm{~ms}$ (Figure 3 ). There were no significant color gradations of CARTO mapping between the RV outflow and RV apex.

Demographic and clinical data of patients with QRS duration longer or shorter than 160 ms during RV apical pacing are shown in Table 1. In univariate logistic regression analysis, patients with QRS duration longer than 160 ms during RV apical pacing were more likely to be male [odds ratio $(\mathrm{OR})=2.31 ; 95 \%$ confidence interval (CI), 1.28-4.15, $\mathrm{p}=0.005]$ and to have longer $\mathrm{QRS}$ duration at baseline (OR=1.02; 95\% CI, 1.06-1.16, $\mathrm{p}<0.001)$, atrial fibrillation $(\mathrm{OR}=2.32 ; 95 \% \mathrm{CI}, 0.95-5.70, \mathrm{p}=0.065)$, hypertension $(\mathrm{OR}=2.26 ; 95 \% \mathrm{CI}, 1.28$ $3.99, \mathrm{p}=0.005)$, heart failure $(\mathrm{OR}=2.12 ; 95 \% \mathrm{CI}, 0.97-4.94, \mathrm{p}=0.061)$, lower LVEF $(\mathrm{OR}=0.98 ; 95 \% \mathrm{CI}, 0.95-$ 1.00, $\mathrm{p}=0.048)$, larger LVEDV $(\mathrm{OR}=1.02 ; 95 \% \mathrm{CI}, 1.01-1.03, \mathrm{p}<0.001)$, larger LVESV $(\mathrm{OR}=1.03 ; 95 \% \mathrm{CI}$ 1.01-1.04, $\mathrm{p}<0.001$ ), higher IVST $(\mathrm{OR}=1.12 ; 95 \% \mathrm{CI} 0.98-1.27, \mathrm{p}=0.088)$, higher LAD $(\mathrm{OR}=1.14 ; 95 \% \mathrm{CI}$ $1.08-1.20, \mathrm{p}<0.001)$, higher $\mathrm{E} / \mathrm{e}^{\prime}(\mathrm{OR}=1.13 ; 95 \% \mathrm{CI} 1.07-1.19, \mathrm{p}<0.001)$, and beta-blocker prescription $(\mathrm{OR}=2.12 ; 95 \% \mathrm{CI} 1.19-3.80, \mathrm{p}=0.061)$.

To avoid statistical coupling of variance, separate multivariate models were constructed that excluded coupled parameters (e.g., two separate models were created for LVEDV and LVESV). The variance inflation factors of LVEDV and LVESV were 5.47 and 5.12, respectively. A multivariate model excluding LVESV showed marginally better c-statistics. A logistic regression multivariate model combining male sex, baseline QRS duration, atrial fibrillation, hypertension, heart failure, LVEF, LVEDV, IVST, LAD, E/e', and use of beta blockers was tested and revealed that baseline QRS duration $(\mathrm{OR}=1.24 ; 95 \% \mathrm{CI} 1.15-1.33, \mathrm{p}<0.01)$, IVST $(\mathrm{OR}=1.20 ; 95 \% \mathrm{CI} 1.02-1.40, \mathrm{p}=0.025), \mathrm{LAD}(\mathrm{OR}=1.08 ; 95 \% \mathrm{CI} 1.01-1.16, \mathrm{p}=0.024)$, and $\mathrm{E} / \mathrm{e}^{\prime}(\mathrm{OR}=1.23$; $95 \%$ CI 1.12-1.35, $\mathrm{p}<0.01)$ were independently associated with QRS duration> $>160 \mathrm{~ms}$ during RV apical pacing (Table 2). 


\section{Discussion}

In this study, we investigated predictive factors of QRS prolongation during RV pacing. The present findings can be summarized as follows: (1) The QRS duration during RV pacing varied among patients, and there was a significant correlation of QRS duration during pacing between the RV outflow and RV apex in each case, denoting that QRS duration during RV pacing depends on the underlying heart condition, not on RV pacing location. (2) Independent predictive factors for QRS duration longer than $160 \mathrm{~ms}$ during RV apical pacing were longer QRS duration, LAD, IVST, and higher E/e' at baseline. These factors suggested that cardiac hypertrophy or diastolic dysfunction, not lower LVEF and larger LV dimension, were associated with prolonged QRS duration during RV pacing.

RV apical pacing is known to generate non-physiological electrical and mechanical dyssynchrony, ${ }^{1}$ and some patients with permanent RV apical pacing experience adverse effects such as deterioration of heart failure or occurrence of atrial fibrillation. ${ }^{2,3} \mathrm{RV}$ non-apical pacing sites were considered alternative pacing sites to reduce adverse effect. However, a randomized-controlled trial showed that non-apical pacing could not provide a preventive effect on heart failure hospitalization, mortality, and burden of atrial fibrillation. ${ }^{18}$ Delayed contraction at the LV lateral site can be observed during RV pacing irrespective of the pacing site, as RV pacing relies on slow cell to cell conduction of the electrical wavefront. In our study, the difference in QRS duration between RV outflow and RV apical pacing was only 12.5+-10.4 ms, suggesting that selecting a non-apical pacing site in RV to compensate for LV electrical dyssynchrony has large limitations.

Biventricular pacing can prevent delayed LV electrical activation and LV lateral contraction. The BLOCK HF trial showed that biventricular pacing reduced the rate of heart failure, hospitalization, and mortality compared to conventional RV pacing in patients with atrioventricular block, NYHA class I, II, or III heart failure, and LVEF of $50 \%$ or lower. ${ }^{19}$ Recent guidelines have recommended biventricular pacing rather than conventional RV pacing for patients with systolic heart failure who depend on chronic pacing. ${ }^{20}$ However, the survival curve of the primary outcome in the BLOCK HF trial showed steady decline even in the biventricular pacing group, suggesting that even patients without systolic heart failure can develop new-onset heart failure. Conflicting preliminary data were reported by the Biventricular Pacing for Atrioventricular Block to Prevent Cardiac Desynchronization Trial investigators. ${ }^{21}$ Complications associated with the implantation of biventricular pacing and high financial burden for implantation make following the guidelines complicated. In the BLOCK HF trial, $10.3 \%$ of patients experienced events related to procedure and device. Moreover, $4.9 \%$ of patients had serious adverse events related to biventricular pacing within 6 months. Additionally, biventricular pacing implantation requires longer fluoroscopic times and, usually, the use of contrast agents to define the coronary venous anatomy. Biventricular pacing actually accounts for less than $10 \%$ of implants in the United States. ${ }^{22}$ Recently, His bundle pacing or left bundle branch pacing has emerged as physiologic pacing managements. ${ }^{4}$ Although several studies have already shown that physiological pacing is superior to conventional RV pacing, not many patients receive this management owing to procedural difficulties, high pacing threshold, and complications. ${ }^{7-9}$ Therefore, traditional RV pacing, in which myocardial capture is achievable in virtually all patients, is still common in clinical practice. We need to identify which patients should receive physiological pacing management.

The QRS duration represents the time interval required for the whole ventricle to be activated. A prolonged QRS duration indicates high risk of morbidity and mortality. ${ }^{11}$ The baseline QRS duration has been used as an indication for CRT as a representative for LV electrical dyssynchrony, and electrical resynchronization reflected by QRS narrowing is valuable to predict the CRT response. ${ }^{12} \mathrm{~A}$ recent study showed that focusing on narrowing QRS by intervening on the level of specialized conduction with sequential LV pacing in peripheral myocardial areas activated later is associated with a better CRT response. ${ }^{14}$ Therefore, the QRS duration represents the degree of electrical dyssynchrony. A prolonged QRS duration during RV pacing was also reported to constitute high risk for heart failure events. ${ }^{15}$ However, the underlying mechanism why some patients show prolonged QRS duration during RV pacing remains to be elucidated.

In our study, independent predictive factors for QRS duration longer than $160 \mathrm{~ms}$ during RV apical pacing were baseline longer QRS duration, LAD, IVST, and higher E/e'. A longer baseline QRS duration indicates 
slight intraventricular conductional disturbances. High IVST represents cardiac hypertrophy, and high LAD and E/e' are widely used as signs of cardiac diastolic failure. ${ }^{23}$ These factors indicate that acquired cardiac disease such as diastolic dysfunction and hypertrophy, not dilated ventricular dimension or lower systolic function, are major determinants of prolonged QRS duration during RV pacing. A recent study ${ }^{24}$ showed that LV size (i.e., LVEDV or LVESV) impacts prolongation of QRS duration during RV pacing. In this study, the LV site was not a significant independent factor but showed a trend to prolong QRS duration during RV pacing. This discrepancy is attributed to the study design. Prior studies did not include echocardiographic factors such as IVST, PWT, and E/e' in the multivariate analysis. Moreover, patients with bundle branch block were included. We speculate that electrical delay due to the effect of hypertrophy or diastolic dysfunction surpasses spatial delay due to the LV dimension to cause QRS prolongation. Fang et al reported that RV apical pacing deteriorated cardiac function particularly in patients with pre-existing LV diastolic dysfunction. ${ }^{25}$ Another study showed that reduced cardiac function following atrioventricular ablation and RV pacing was more often observed in patients with numerous comorbidities. ${ }^{26,27}$ Conversely, in patients with congenital atrioventricular block, long-term RV pacing did not develop into heart failure. ${ }^{28}$

Longer QRS duration is a sign of electrical dyssynchrony, which can cause reduction of cardiac function. A prior study showed an association between longer QRS duration of premature ventricular contractions (PVC) and reduced cardiac function (i.e., PVC-induced cardiomyopathy). ${ }^{29}$ QRS duration of PVC, not the PVC origin or baseline LVEF, was found to be an independent predictor for the recovery of LV function after ablation for PVC. ${ }^{30}$ This finding is compatible with our results, i.e., not the origin, but the underlying heart disease, which caused electrical activation delay, strongly affected cardiac function. ${ }^{31,32}$

Study limitations

In this study, we enrolled patients planning to undergo radiofrequency ablation, not pacemaker implantation. The results may be limited by inclusion of a heterogeneous population. The potential for residual confounding should be taken into account when interpreting data. However, the aim of this study was to identify factors prolonging QRS duration during RV pacing, and the average age or underlying comorbidities of our patients were similar with those of patients planning to undergo pacemaker implantation in another study. ${ }^{15}$

In our study, pure selective His bundle or right bundle branch capture was not observed during pacing around the His or right bundle branch area. We planned to exclude QRS measurement when pacing purely captured the His bundle or right bundle branch. In attempting non-apical RV pacing, His bundle or right bundle branch capture was accidentally observed in a few patients. However, the incidence was exceedingly rare, and pacing on these structures has already been reported to be superior to conventional RV pacing. ${ }^{33}$ To examine the effect of delayed activation not via the His bundle or right bundle branch, data associated with narrowing QRS duration by RV pacing were excluded from this study.

The selection of RV outflow and RV apex might depend on the discretion of the operators. However, the general area of the RV outflow and RV apex can be identified by 3D mapping, and the pacing electrical axis (the inferior and superior axes signify the RV outflow and RV apical pacing, respectively) can verify the location of the RV outflow and RV apex. The RV pacing site is defined by both fluoroscopic imaging and the electrocardiographic axis in clinical practice.

Our study was also limited by its single-center design and small study population. Further multicenter studies including pacemaker implantation with long-term follow-up and large numbers of patients are warranted to confirm these findings.

Conclusion

The QRS duration during RV pacing depends on the underlying heart disease, not on the RV pacing site. Cardiac hypertrophy and diastolic LV dysfunction prolong electrical activation from the RV to LV lateral sites. Physiological pacing management should be considered for patients with obvious cardiac hypertrophy and diastolic LV dysfunction.

Acknowledgements 
The authors thank Noboru Kitamura and Takeru Takada for technical assistance and support during the pacing study.

References

1. Tops LF, Suffoletto MS, Bleeker GB, et al. Speckle-tracking radial strain reveals left ventricular dyssynchrony in patients with permanent right ventricular pacing. J Am Coll Cardiol 2007;50:1180-1188.

2. Wilkoff BL, Cook JR, Epstein AE, et al. Dual-chamber pacing or ventricular backup pacing in patients with an implantable defibrillator: the dual chamber and VVI implantable defibrillator (DAVID) trial. JAMA 2002;288:3115-3123.

3. Nahlawi M, Waligora M, Spies SM, Bonow RO, Kadish AH, Goldberger JJ. Left ventricular function during and after right ventricular pacing. J Am Coll Cardiol 2004;44:1883-1888.

4. Vijayaraman P, Chung MK, Dandamudi G, et al. His bundle pacing. J Am Coll Cardiol 2018;72:927-947.

5. Li Y, Chen K, Dai Y, et al. Left bundle branch pacing for symptomatic bradycardia: implant success rate, safety, and pacing characteristics. Heart Rhythm 2019;16:1758-1765.

6. Zhang S, Zhou X, Gold MR. Left bundle branch pacing: JACC review topic of the week. J Am Coll Cardiol 2019;74:3039-3049.

7. Sharma PS, Dandamudi G, Naperkowski A, et al. Permanent His-bundle pacing is feasible, safe, and superior to right ventricular pacing in routine clinical practice. Heart Rhythm 2015;12:305-312.

8. Vijayaraman P, Dandamudi G, Zanon F, et al. Permanent His bundle pacing: recommendations from a multicenter His Bundle Pacing Collaborative Working Group for standardization of definitions, implant measurements, and follow-up. Heart Rhythm 2018;15:460-468.

9. Lustgarten DL, Sharma PS, Vijayaraman P. Troubleshooting and programming considerations for His bundle pacing. Heart Rhythm 2019;16:654-662.

10. Dreger H, Maethner K, Bondke H, Baumann G, Melzer C. Pacing-induced cardiomyopathy in patients with right ventricular stimulation for $>15$ years. Europace 2012;14:238-242.

11. Iuliano S, Fisher SG, Karasik PE, Fletcher RD, Singh SN, Department of Veterans Affairs Survival Trial of Antiarrhythmic Therapy in Congestive Heart Failure. QRS duration and mortality in patients with congestive heart failure. Am Heart J 2002;143:1085-1091.

12. Hsing JM, Selzman KA, Leclercq C, et al. Paced left ventricular QRS width and ECG parameters predict outcomes after cardiac resynchronization therapy: PROSPECT-ECG substudy. Circ Arrhythm Electrophysiol 2011;4:851-857.

13. Iler MA, Hu T, Ayyagari S, et al. Prognostic value of electrocardiographic measurements before and after cardiac resynchronization device implantation in patients with heart failure due to ischemic or nonischemic cardiomyopathy. Am J Cardiol 2008;101:359-363.

14. Trucco E, Tolosana JM, Arbelo E, et al. Improvement of reverse remodeling using electrocardiogram fusion-optimized intervals in cardiac resynchronization therapy: a randomized study. JACC Clin Electrophysiol 2018;4:181-189.

15. Chen S, Yin Y, Lan X, et al. Paced QRS duration as a predictor for clinical heart failure events during right ventricular apical pacing in patients with idiopathic complete atrioventricular block: results from an observational cohort study (PREDICT-HF). Eur J Heart Fail 2013;15:352-359.

16. Shukla HH, Hellkamp AS, James EA, et al. Heart failure hospitalization is more common in pacemaker patients with sinus node dysfunction and a prolonged paced QRS duration. Heart Rhythm 2005;2:245-251. 
17. Khurshid S, Liang JJ, Owens A, et al. Longer paced QRS duration is associated with increased prevalence of right ventricular pacing-induced cardiomyopathy. J Cardiovasc Electrophysiol 2016;27:1174-1179.

18. Kaye GC, Linker NJ, Marwick TH, et al. Effect of right ventricular pacing lead site on left ventricular function in patients with high-grade atrioventricular block: results of the Protect-Pace Study. Eur Heart J $2015 ; 36: 856-862$.

19. Curtis AB, Worley SJ, Adamson PB, et al. Biventricular pacing for atrioventricular block and systolic dysfunction. N Engl J Med 2013;368:1585-1593.

20. Normand C, Linde C, Singh J, Dickstein K. Indications for cardiac resynchronization therapy: a comparison of the major international guidelines. JACC Heart Fail 2018;6:308-316.

21. Beck H, Curtis AB. Right ventricular versus biventricular pacing for heart failure and atrioventricular block. Curr Heart Fail Rep 2016;13:230-236.

22. Greenspon AJ, Patel JD, Lau E, et al. Trends in permanent pacemaker implantation in the United States from 1993 to 2009: increasing complexity of patients and procedures. J Am Coll Cardiol 2012;60:1540-1545.

23. Redfield MM. Heart failure with preserved ejection fraction. N Engl J Med 2016;375:1868-1877.

24. Zweerink A, Bakelants E, Stettler C, Burri H. His bundle pacing to avoid electrical dyssynchrony with traditional right ventricular pacing: importance of heart size. Int J Cardiol 2020;311:54-57.

25. Fang F, Zhang Q, Chan JY, et al. Deleterious effect of right ventricular apical pacing on left ventricular diastolic function and the impact of pre-existing diastolic disease. Eur Heart J 2011;32:1891-1899.

26. Kiehl EL, Makki T, Kumar R, et al. Incidence and predictors of right ventricular pacing-induced cardiomyopathy in patients with complete atrioventricular block and preserved left ventricular systolic function. Heart Rhythm 2016;13:2272-2278.

27. Chen L, Hodge D, Jahangir A, et al. Preserved left ventricular ejection fraction following atrioventricular junction ablation and pacing for atrial fibrillation. J Cardiovasc Electrophysiol 2008;19:19-27.

28. Sagar S, Shen WK, Asirvatham SJ, et al. Effect of long-term right ventricular pacing in young adults with structurally normal heart. Circulation 2010;121:1698-1705.

29. Del Carpio Munoz F, Syed FF, Noheria A, et al. Characteristics of premature ventricular complexes as correlates of reduced left ventricular systolic function: study of the burden, duration, coupling interval, morphology and site of origin of PVCs. J Cardiovasc Electrophysiol 2011;22:791-798.

30. Deyell MW, Park KM, Han Y, et al. Predictors of recovery of left ventricular dysfunction after ablation of frequent ventricular premature depolarizations. Heart Rhythm 2012;9:1465-1472.

31. Walters TE, Rahmutula D, Szilagyi J, et al. Left ventricular dyssynchrony predicts the cardiomyopathy associated with premature ventricular contractions. J Am Coll Cardiol 2018;72:2870-2882.

32. Huizar JF, Ellenbogen KA, Tan AY, Kaszala K. Arrhythmia-induced cardiomyopathy: JACC state-ofthe-art review. J Am Coll Cardiol 2019;73:2328-2344.

33. Arnold AD, Shun-Shin MJ, Keene D, et al. His resynchronization versus biventricular pacing in patients with heart failure and left bundle branch block. J Am Coll Cardiol 2018;72:3112-3122.

Table 1. Baseline patient characteristics

\begin{tabular}{lllll}
\hline & & QRS duration $>$ & QRS duration $<$ & \\
Characteristics & Total $(\mathrm{N}=211)$ & $160 \mathrm{~ms}(\mathrm{~N}=82)$ & $160 \mathrm{~ms}(\mathrm{~N}=129)$ & p-value \\
\hline Male sex & $124(58.8)$ & $58(70.7)$ & $66(51.2)$ & 0.006 \\
Age (years) & $66 \pm 12$ & $67 \pm 10$ & $66 \pm 13$ & 0.287
\end{tabular}




\begin{tabular}{lllll}
\hline Characteristics & Total $(\mathrm{N}=211)$ & $\begin{array}{l}\text { QRS duration }> \\
160 \mathrm{~ms}(\mathrm{~N}=82)\end{array}$ & $\begin{array}{l}\text { QRS duration }< \\
160 \mathrm{~ms}(\mathrm{~N}=129)\end{array}$ & p-value \\
\hline $\begin{array}{l}\text { Baseline ECG } \\
(\mathrm{ms})\end{array}$ & $85.0 \pm 7.5$ & $88.2 \pm 7.5$ & $83.0 \pm 6.8$ & $<0.01$ \\
Atrial fibrillation & $181(85.8)$ & $75(91.5)$ & $106(82.2)$ & 0.007 \\
Hypertension & $108(51.2)$ & $52(63.4)$ & $56(43.4)$ & 0.005 \\
Heart Failure & $27(12.8)$ & $15(18.3)$ & $12(9.3)$ & 0.089 \\
Ischemic & $11(5.2)$ & $6(7.3)$ & $5(3.9)$ & 0.344 \\
cardiomyopathy & & & & \\
LVEF (\%) & $61.7 \pm 10.6$ & $59.9 \pm 12.2$ & $62.9 \pm 9.3$ & 0.055 \\
LVEDV (ml) & $106.8 \pm 35.0$ & $121.7 \pm 39.8$ & $97.4 \pm 27.9$ & $<0.01$ \\
LVESV (ml) & $43.0 \pm 26.2$ & $51.9 \pm 34.1$ & $37.3 \pm 17.4$ & 0.01 \\
IVST (mm) & $10.1 \pm 2.7$ & $10.6 \pm 2.2$ & $9.8 \pm 3.0$ & 0.046 \\
PWT (mm) & $10.1 \pm 6.8$ & $10.0 \pm 1.6$ & $10.2 \pm 8.6$ & 0.782 \\
LAD (mm) & $38.3 \pm 7.3$ & $42.1 \pm 6.1$ & $36.7 \pm 7.2$ & $<0.01$ \\
E/e & $13.2 \pm 6.5$ & $15.9 \pm 7.6$ & $11.5 \pm 4.9$ & $<0.01$ \\
$\beta$ blocker use & $73(34.6)$ & $37(45.1)$ & $36(27.9)$ & 0.012 \\
\hline
\end{tabular}

Data are presented as $\mathrm{n}(\%)$ and mean $\pm \mathrm{SD}$.

$\mathrm{ECG}=$ electrocardiogram, IVST $=$ interventricular septum thickness, LAD = left atrial dimension, LVEDV $=$ left ventricular end-diastolic volume; $\mathrm{LVEF}=$ left ventricular ejection fraction; LVESV = left ventricular end-systolic volume; PWT = posterior left ventricular wall thickness

Table 2. Univariate and multivariate logistic regression analyses to predict wide QRS duration during RV pacing

\begin{tabular}{lllllll}
\hline & Univariate & Univariate & Univariate & Multivariate & Multivariate & Multivariate \\
\hline & OR & $95 \%$ CI & $\mathrm{p}$ Value & OR & $95 \%$ CI & p Value \\
Male sex & 2.31 & $1.28-4.15$ & 0.005 & 1.05 & $0.43-2.56$ & 0.91 \\
Age & 1.01 & $0.99-1.04$ & 0.31 & & & \\
Baseline QRS & 1.02 & $1.06-1.16$ & $<0.001$ & 1.24 & $1.15-1.33$ & $<0.001$ \\
Atrial fibrillation & 2.32 & $0.95-5.70$ & 0.065 & 0.63 & $0.12-2.14$ & 0.46 \\
Hypertension & 2.26 & $1.28-3.99$ & 0.005 & 1.84 & $0.77-4.39$ & 0.17 \\
Heart Failure & 2.12 & $0.97-4.94$ & 0.061 & 0.76 & $0.23-2.58$ & 0.66 \\
Ischemic cardiomyopathy & 1.96 & $0.58-6.64$ & 0.29 & & & \\
LVEF & 0.98 & $0.95-1.00$ & 0.048 & 1.00 & $0.98-1.01$ & 0.77 \\
LVEDV & 1.02 & $1.01-1.03$ & $<0.001$ & 1.01 & $1.00-1.02$ & 0.15 \\
LVESV & 1.03 & $1.01-1.04$ & $<0.001$ & & & \\
IVST & 1.12 & $0.98-1.27$ & 0.088 & 1.20 & $1.02-1.40$ & 0.025 \\
PWT & 1.00 & $0.95-1.04$ & 0.82 & & & \\
LAD & 1.14 & $1.08-1.20$ & $<0.001$ & 1.08 & $1.01-1.16$ & 0.024 \\
E/e & 1.13 & $1.07-1.19$ & $<0.001$ & 1.23 & $1.12-1.35$ & $<0.001$ \\
$\beta$ blocker use & 2.12 & $1.19-3.80$ & 0.011 & 0.70 & $0.30-1.65$ & 0.42 \\
\hline
\end{tabular}

$\mathrm{CI}=$ confidence interval, IVST $=$ interventricular septum thickness, LAD $=$ left atrial dimension, LVEDV $=$ left ventricular end-diastolic volume, $\mathrm{LVEF}=$ left ventricular ejection fraction, $\mathrm{LVESV}=$ left ventricular end-systolic volume, $\mathrm{OR}=$ odds ratio, $\mathrm{PWT}=$ posterior left ventricular wall thickness

Figure 1 


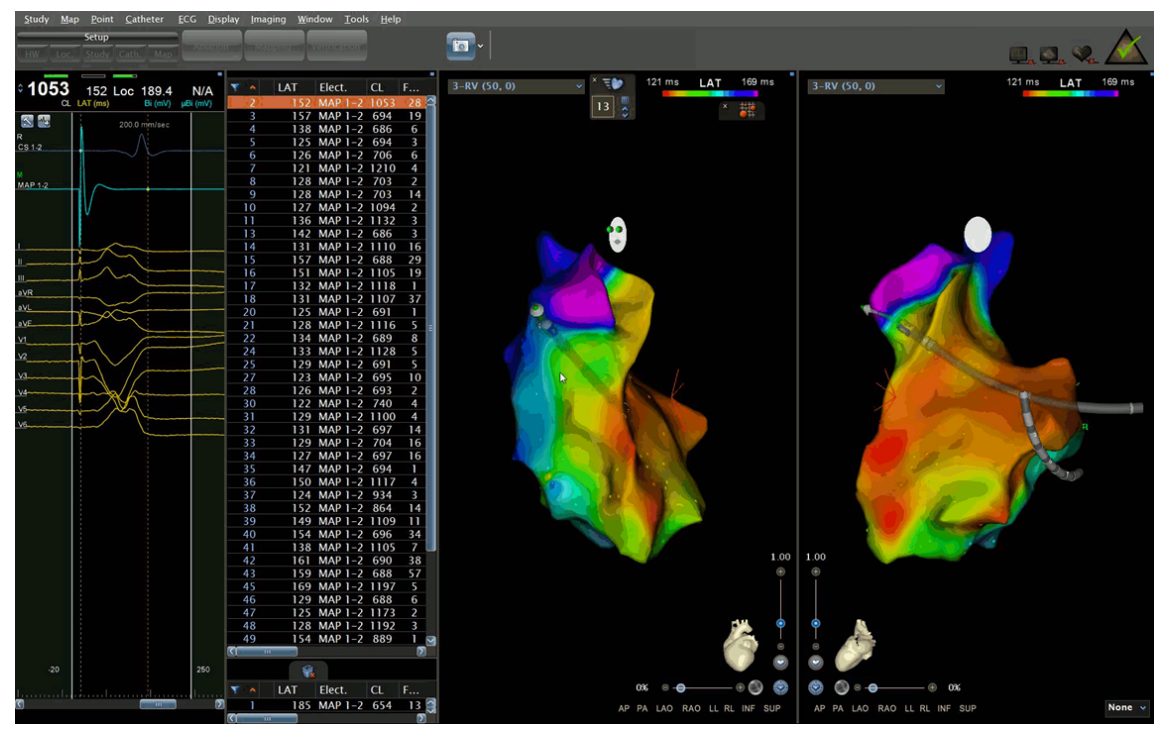

Creation of paced QRS (pQRS) mapping in the right ventricle. The initial timing of QRS duration is set to the pacing artifact signal. The pQRS duration is color coded and superimposed on the anatomic map.

Figure 2

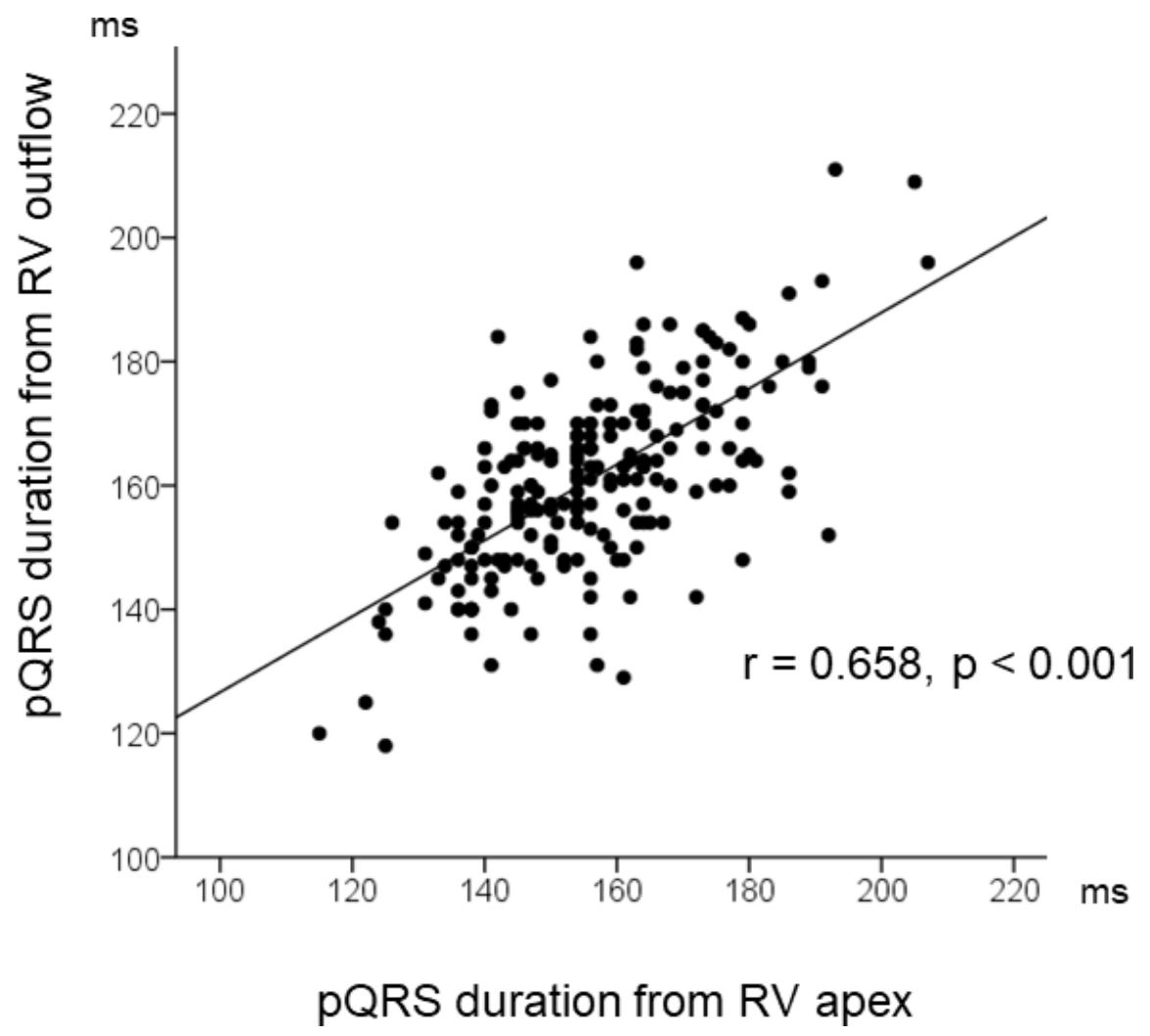

Relationship of paced QRS (pQRS) duration between the right ventricular (RV) outflow and apex.

Figure 3 

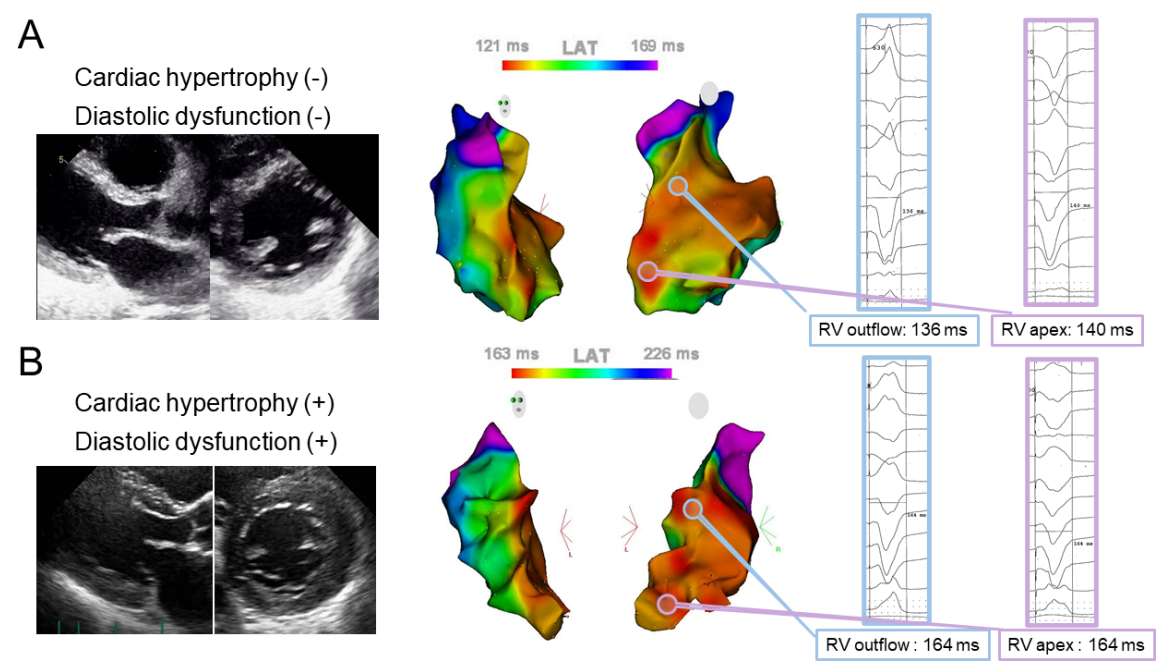

Echocardiography, paced QRS map, and QRS duration during right ventricular (RV) apex and RV outflow pacing are shown in a patient without left ventricular (LV) hypertrophy and diastolic dysfunction (A) and with LV hypertrophy and diastolic dysfunction (B). QRS duration during RV pacing depends on the underlying heart disease, not the pacing site. 